\title{
A Study on Channel Capacity Enhancement using Massive MIMO Systems
}

\author{
Dr. Dilip Sharma ${ }^{1}$ and Nidhi choudhary ${ }^{2}$ \\ ${ }^{1}$ Faculty, UEC, India. drdilipsharma72@gmail.com \\ ${ }^{2} \mathrm{PG}$ student, UEC, India. Choudharynidhi015@gmail.com
}

DOI $<$ 10.26821/IJSHRE.8.2.2020.8205>

\begin{abstract}
Wireless Communication Systems are facing the challenges regarding increasing number of users, limited bandwidth and a continuous need for high data rates. One of the fundamental mechanisms to enhance channel capacity is the use of Multiple Input-Multiple Output (MIMO) systems for channel capacity enhancement. This paper presents review on the various implementation mechanisms of MIMO-Systems specially focusing on Space Time Block Coding (STBC). A brief introduction pertaining to the basics of MIMO systems have also been put forth for the ease of understanding of MIMO based systems.
\end{abstract}

Keywords- BER, STBC, OFDM, and MIMO

\section{INTRODUCTION}

In wireless communication, the receiver side BER strongly affected by channels noise, interference, distortion, synchronization error and wireless multipath fading channels, Multiple-input and multiple-output( MIMO) systems have resulted in higher spectral efficiency and capacity [1]. MIMO communications, the system is equipped with multiple antennas at both the transmitter and the receiver technique [2]. The multiple antenna scheme gives a more reliable performance through array gain, diversity and spatial multiplexing [3]. These concepts are briefly discussed below.

The growing demand of multimedia services and the progress of Internet related contents lead to increasing interest to high speed communications network [4]. The requirement for flexibility and wide bandwidth imposes the use of efficient transmission systems that would fit to the characteristics of wideband channels especially in wireless environment where the channel is very challenging process [5]. In wireless environment the signal is propagating since the transmitter to the receiver along number of different paths, collectively referred as multipath communication. While propagating the signal power drops of due to the following effects: a path loss, macroscopic fading and microscopic fading. The fading of the signal can be mitigated by different diversity methods [6].

\section{LITERATURE SURVEY}

J Mo, P Schniter et al. proposed the technique for Channel estimation in broadband millimeter wave MIMO systems. They used the concept of one bit analog to digital converters. They showed that that the cannel estimation in MIMO systems was a tedious task and hence needed an effective technique for accuracy. The broadband millimeter MIMO systems were used. It was shown that wireless channels were random in nature and hence they needed proper channel estimation [1].

J Rodríguez-Fernández et al. proposed the Frequency-Domain Compressive Channel Estimation for channels that showed frequency selective nature. As it is prevalent with wireless channels, the compressive sensing for MIMO channels is necessary. Hence compressive sensing for multi-user mechanism was critical in channel sensing for MIMO based systems [2].

Prabina Pattanayak et al. proposed a technique for evaluating the Bit Error Rate or BER of MIMO-STBC systems. It was shown that Space time block coding also known as STBC was an effective

Dr. Dilip Sharma and Nidhi choudhary, Vol 8 Issue 2, pp 52-57, February 2020 
Volume 8 Issue 2 February 2020

technique for practically implementing MIMO systems with streams of digital data [3].

Y Li et al. proposed the scheme for massive MIMO systems. It was shown that massive MIMO was a combination of several transmitters and receivers and that it had a significantly indelible effect on the channel capacity. Massive MIMO when compared to simple MIMO was much more effective in enhancing the spectral efficiency of the system [4].

S Park et al. proposed Dynamic subarrays for hybrid precoding in case MIMO systems were used for frequency selective channels. In the system, estimation for channels showed the channels non-ideal nature. As it is prevalent with wireless channels, the compressive sensing for MIMO channels is necessary [5].

C Chen et al. proposed an Adaptive fuzzy asymptotic control of MIMO systems. This used the concepts of artificial neural networks in conjugation with fuzzy logic for the control of MIMO systems. The control mechanism was employed with using the fuzzy controller to take advantage of the non-clear demarcation of boundaries or states separated by a clear boundary value [6].

X Gao et al. proposed Energy-efficient hybrid analog and digital precoding for massive MIMO systems. It was shown that the proposed system performed better compared to the conventional V-Blast techniques used for mmwave MIMO systems with multiple transmitters and receivers. MIMO pre-coding helped in the channel capacity to increase and thereby increases the spectral efficiency [7].

X Yu et al. proposed Alternating Minimization Algorithms for Hybrid Precoding for Massive MIMO systems. This included the cyclic lattice reduction (CLR) algorithm in which the alternative code segment is generated for MIMO encoding. The pre-coding helped in the channel capacity to increase and thereby increases the spectral efficiency [8].

J Mo et al. proposed increasing the capacity of the system by harnessing the channel state information (CSI) of the channel. In this approach the channel is sensed by channel sounding mechanism. The technique is so designed to find the state of the channel. The major challenge in this system is the abrupt nature of wireless channels. The need for equalization stems from the fact that channels behave differently for different paths and frequencies [9].

A Pitarokoilis proposed a system utilizing the maximum ratio combining (MRC) algorithm for time critical applications. In this case the MIMO system used a massive MIMO structure with the successive signal detection algorithm. This was particularly suited to the downlink MIMO approach [10].

\section{MIMO SYSTEM MODEL}

The basics of the MIMO system model are discussed below.

\section{Array Gain}

In MIMO communications, array gain is the average increase in the SNR at the receiver that occurs from the coherent combining effect of the multiple antennas at the transmitter or receiver or both. Usually, multi antenna systems require a perfect knowledge of the channel either at the receiver or the transmitter or both to achieve an array gain. It is mathematically given by:

$$
A G=\frac{S N R_{M I M O}}{S N R_{S I S O}}
$$

Here,

AG represents Array Gain

SNR stands for Signal to Noise Ratio

MIMO stands for multiple input multiple output

SISO stands for single input single output

Transmitter Array Gain: If the channel is known to the transmitter with multiple antennas, the transmitter will weigh the transmission with weights, depending on the channel coefficients, so that there is coherent combining at one antenna receiver. This is the MISO case. This type of array gain is called transmitter array gain. Mathematically, if the combined transmitted signal is given by:

$$
S_{T X}=\sum_{i=1}^{i=n} S_{i} W_{i}
$$

then the following ratio is called the transmitter array gain

$$
A G_{T X}=\frac{S_{T X M I M O}}{S_{T X ~ S I S O}}
$$

Here,

AG represents array gain 
Volume 8 Issue 2 February 2020

$S_{T X}$ SISO represents the transmitted SISO signal

$S_{T X \text { мIMO }}$ represents the transmitted MIMO signal

$W_{i}$ represents the weights of the ' $\mathrm{i}$ ' parallel transmission paths corresponding to the path co-efficient

Receiver Array Gain: If we have a sing antenna at the transmitter and no knowledge of tl channel and a receiver with multiple antennas, whis has perfect knowledge of the channel, then the receiv can suitably weight the incoming signals so that th coherently add up at the output, thereby enhancing t] signal. This is the SIMO case. This type of array gain called receiver array gain. Similarly the receiver arri. gain is given by:

$$
A G_{R X}=\frac{S_{R X \text { MIMO }}}{S_{R X} \text { SISO }}
$$

Here,

AG represents array gain

$S_{R X \text { SISO }}$ represents the transmitted SISO signal represents the transmitted MIMO signal

\section{Diversity Gain}

In MIMO systems, the same information transmitted from multiple transmit antennas and simultaneously received at multiple receive antennas. Since the fading for each link between a pair of transmit and receive antennas usually can be considered to be independent, the probability that the information is detected exactly is increased. Space time codes a designed to exploit following two resources.[11]

$$
\begin{aligned}
& \text { DOF }=\min \left(N_{T}, N_{R}\right) \\
& \text { Diversity }=N_{T}, N_{R}
\end{aligned}
$$

Here,

DOF represents degree of freedom

$N_{T}$ represents number of transmitters

$N_{R}$ represents number of receivers

\section{Multi Antenna Systems}

Multi antenna systems can be classified as single-input multiple-output (SIMO), multiple-input single-output (MISO), and multiple-input multipleoutput (MIMO) systems. In order to develop the inputoutput relations of SIMO, MISO, MIMO systems, we first describe the single-input single-output (SISO) system [14].

\section{Single Input Single Output System}

The schematic block diagram of a SISO system is depicted in Figure 1.

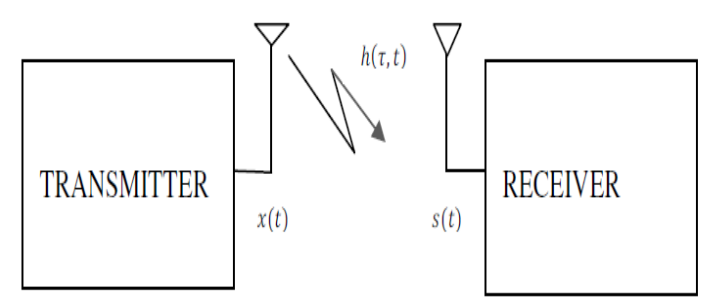

Fig.1: Block diagram of SISO system

The input-output relationship of the SISO system is given by

$\uparrow_{s}(t)=\int_{0}^{\tau t o t a l} h(\tau, t) x(t-\tau) d \tau=h(\tau, t) * x(t)(7)$

Where, $x(t)$ is the transmitted signal, $s(t)$ is the received signal at timet, ttotal is the duration of the impulse response, and denotes the convolution operation

\section{Single Input Multiple Output System}

The schematic block diagram of a SIMO system with single transmit antenna and receive antennas is depicted in Figure 2.

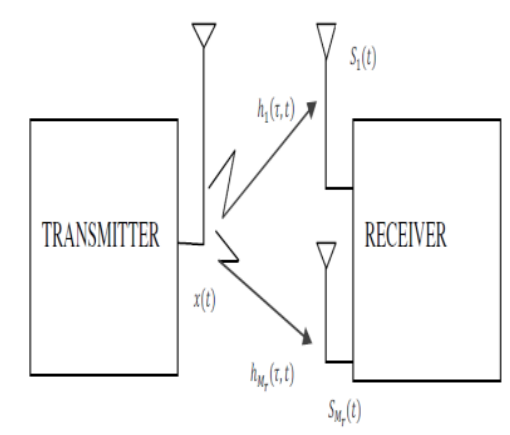

Fig.2: Block diagram of a SIMO system

The received signals at the respective receive antennas are given by

$$
\begin{aligned}
& S_{1}(t)=h_{1}(\tau, t) * x(t) \\
& S_{2}(t)=h_{2}(\tau, t) * x(t) \\
& S_{M r}(t)=h_{M r}(\tau, t) * x(t)
\end{aligned}
$$


Volume 8 Issue 2 February 2020

Therefore, the input-output relationship of a SIMO system can be expressed as

$$
S(t)=H(\tau, t) * x(t)
$$

Where

$S(t)=\left[S_{1}(t) S_{2}(t) \ldots \ldots \ldots S_{M r}(t)\right]^{T}$ in the received signal vector

$h(t)=\left[h_{1}(t) h_{2}(t) \ldots \ldots \ldots h_{M r}(t)\right]^{T}$ in the channel vector

\section{Multipath Environment}

In wireless environment, transmitted signal follow several propagation paths. Many of these paths, having reflected from surrounding objects, reach the receiver with different propagation delays. This multipath leads to delay spread, inter symbol interference (ISI), fading and random phase distortion. Figure 4.4 describes this phenomenon. The corresponding channel impulse response is shown in Figure 3.

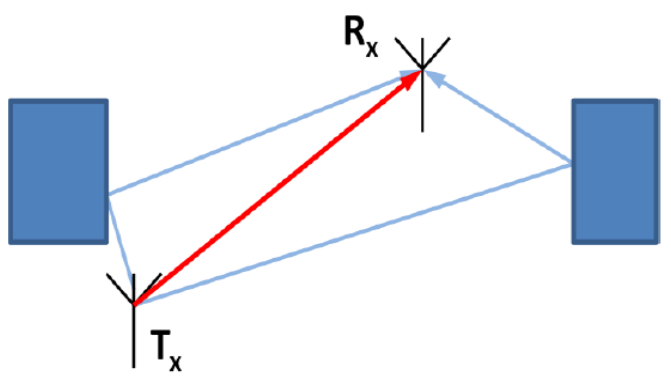

Fig.3: Multipath Environment in Wireless Communications

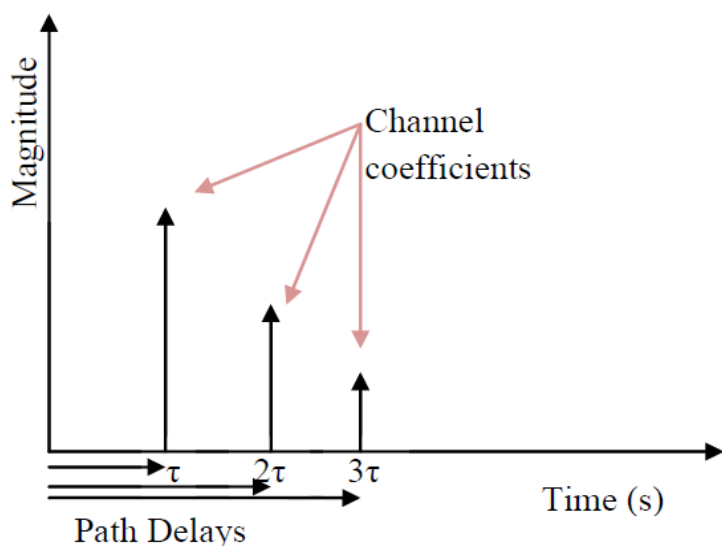

Fig.4: Channel Impulse response in Multipath Environment

Delayed copies of the transmitted signal interfere with subsequent signals, causing Inter Symb
Interference (ISI). Therefore transmitted symbol rate is limited by the delay spread of the channel. Multipath Propagation causes MIMO channels to be frequency selective which cannot have flat frequency response. To combat this effect MIMO is combined with OFDM. OFDM transforms the frequency selective fading channel into parallel flat fading sub channels, but the length of CP inserted should be greater than the channel length.

For a $2 \times 2$ MIMO-OFDM configuration the received OFDM symbols are given the equations 2 . The same thing is represented in matrix form in equation 3. Here the term Xi represents the transmitted symbol from the ith transmitting antenna, the term $\mathrm{Yj}$ represents the received symbol from the $j$ th receiving antenna, the term $\mathrm{Ni}$ represents the noise component present in the ith symbol and the term Hij represents the channel coefficient corresponding to the ith transmitting antenna and jth receiving antenna.

$R_{x}$. Ant 1: $Y_{1}(K)=H_{11}(K) X_{1}(K)+H_{12}(K) X_{2}(K)+$ $N_{1}(K) \quad(12)$

$R_{x}$. Ant 2: $Y_{2}(K)=H_{21}(K) X_{1}(K)+H_{22}(K) X_{2}(K)+$ $N_{2}(K)$

$\left[\begin{array}{l}Y_{1}(K) \\ Y_{2}(K)\end{array}\right]=\left[\begin{array}{ll}H_{11}(K) & H_{12}(K) \\ H_{21}(K) & H_{22}(K)\end{array}\right]\left[\begin{array}{l}X_{1}(K) \\ X_{2}(K)\end{array}\right]+\left[\begin{array}{l}N_{1}(K) \\ N_{2}(K)\end{array}\right]$

In a Direct path environment, where the reflected waves from multiple objects are absent, in channel looks to be flat and contains a single coefficient in its impulse response. This type of channel is can be modeled using Additive White Gaussian Noise (AWGN) channel. The model does not account for the phenomena of fading, interference, frequency selectivity, and nonlinearity or dispersion. In a multipath environment, the channel is always frequency selective type. This type of channel can be modeled using Rayleigh random distribution.

\section{THE STBC ALGORITHM}

STBC stands for space time block coding. The 
Volume 8 Issue 2 February 2020

space-time encoding mapping of Alamouti's two-twobranch transmits diversity technique can be represented by the coding matrix:

Coding matrix:

$$
X_{1}=\left[\begin{array}{cc}
x_{1} & -x_{2}^{*} \\
x_{2} & x_{1}^{*}
\end{array}\right]
$$

In the coding matrix $\mathrm{X} 1$, the subscript index gives the transmit rate compared to a SISO system. For Alamouti's scheme, the transmission rate is 1 . The rows of the coding matrix represent the transmit antennas while its columns correspond to different time instances. It is clear that the encoding is done in both the space and time domains. Let us denote the transmit sequence from antennas one and two by $x^{1}$ and $x^{2}$, respectively.

$$
\begin{aligned}
& x^{t 1}=\left[x_{1},-x *_{2}\right] \\
& x^{t 2}=\left[x_{2}, x *_{1}\right]
\end{aligned}
$$

The key feature of the Alamouti scheme is that the transmit sequences from the two transmit antennas are orthogonal, since the inner product of the sequences $x^{1}$ and $x^{2}$ is zero, i.e.

$$
x^{t 1} \cdot x^{t 2}=x_{1} x *_{2}-x *_{2} x_{1}
$$

The code matrix has the following property:

$$
\begin{aligned}
X . X^{H} & =\left[\begin{array}{cc}
\left|x_{1}\right|^{2}+\left|x_{2}\right|^{2} & 0 \\
0 & \left|x_{1}\right|^{2}+\left|x_{2}\right|^{2}
\end{array}\right] \\
& =\left(\left|x_{1}\right|^{2}+\left|x_{2}\right|^{2}\right) I_{2}
\end{aligned}
$$

here $l_{2}$ is a $2 \times 2$ identity matrix

At the receive antenna, the received signals over two consecutive symbol periods, denoted by $r_{1} \mathrm{And} r_{2}$ for time $\mathrm{t}$ and $t+T$, respectively, can be expressed as

$$
\begin{aligned}
& r_{1}=h_{1} x_{1}+h_{2} x_{2}+n_{1} \\
& r_{2}=-h_{1} x *_{1}+h_{2} x *_{2}+n_{2}
\end{aligned}
$$

Where $n_{1}$ and $n_{2}$ are independent complex variables with zero mean and power spectral density $N 0 / 2$ per dimension, representing additive white Gaussian noise samples at time $t$ and $t+T$, respectively.

\section{CONCLUSION}

It can be concluded that MIMO systems and moreover massive MIMO systems are the only way out to provide limited bandwidth yet bandwidth hungry applications. Massive MIMO may serve as a technique to increase the system capacity and date rate. This paper proposes the basics review of MIMO systems and their merits and applications.

\section{REFRENCES}

[1] J Mo, P Schniter, RW Heath, "Channel estimation in broadband millimeter wave MIMO systems with few-bit ADCs", IEEE 2018

[2] J Rodríguez-Fernández, "Frequency-Domain Compressive Channel Estimation for FrequencySelective Hybrid Millimeter Wave MIMO Systems", IEEE 2018

[3] Prabina Pattanayak, Vinay Kumar Trivedi, Sayan Chakraborty, Preetam Kumar, "BER Performance of Multi User Scheduling for MIMO-STBC and MIMOOFDM Broadcast Network with Imperfect CSI", IEEE 2017

[4] Y Li, C Tao, G Seco-Granados, "Channel estimation and performance analysis of one-bit massive MIMO systems", IEEE 2017

[5] S Park, A Alkhateeb, RW Heath, "Dynamic subarrays for hybrid precoding in wideband mmWave MIMO systems", IEEE 2017

[6] C Chen, Z Liu, K Xie, Y Liu, Y Zhang, “Adaptive fuzzy asymptotic control of MIMO systems with unknown input coefficients via a robust Nussbaum gainbased approach", IEEE 2017

[7] X Gao, L Dai, S Han, I Chih-Lin, "Energy-efficient hybrid analog and digital precoding for mmWave MIMO systems with large antenna arrays”, IEEE 2016

[8] X Yu, JC Shen, J Zhang, “Alternating Minimization Algorithms for Hybrid Precoding in Millimeter Wave MIMO Systems", IEEE 2016

[9] J Mo, RW Heath, "Capacity analysis of one-bit quantized MIMO systems with transmitter channel state information", IEEE 2015

[10] A Pitarokoilis, SK Mohammed, "Uplink performance of time-reversal MRC in massive MIMO systems subject to phase noise", IEEE 2015

[11] EG Larsson, O Edfors, F Tufvesson, "Massive MIMO for next generation wireless systems", IEEE 2014

[12] E Björnson, J Hoydis, M Kountouris, "Massive MIMO systems with non-ideal hardware: Energy efficiency, estimation, and capacity limits”, IEEE 2014

[13] HQ Ngo, EG Larsson, "Energy and spectral efficiency of very large multiuser MIMO systems", IEEE 2013

[14] X Chen, X Wang, X Chen, "Energy-efficient optimization for wireless information and power transfer in large-scale MIMO systems employing energy beam- 
ijournals: International Journal of Software \& Hardware Research in Engineering

ISSN-2347-4890

Volume 8 Issue 2 February 2020

forming", IEEE 2013

[15] O El Ayach, RW Heath, S Abu-Surra, "Low

complexity precoding for large millimeter wave MIMO

systems", IEEE 2012 